\title{
Introduction to the Special Issue: Futuristic trends and emergence of technology in biomedical, nonlinear dynamics and control engineering
}

\author{
Gennady Veselov ${ }^{1}$, Alexey Tselykh ${ }^{2}$, Ashutosh Sharma ${ }^{3}$ \\ Institute of Computer Technology and Information Security, Southern Federal University, \\ Rastov-on-Don, Russia \\ ${ }^{3}$ Corresponding author \\ E-mail: ${ }^{1}$ gev@sfedu.ru, ${ }^{2}$ tselykh@sfedu.ru, ${ }^{3}$ ashutosh@sfedu.ru
}

Accepted 28 September 2021

DOI https://doi.org/10.21595/jve.2021.22226

Check for updates

Copyright (C) 2021 Gennady Veselov, et al. This is an open access article distributed under the Creative Commons Attribution License, which permits unrestricted use, distribution, and reproduction in any medium, provided the original work is properly cited.

\begin{abstract}
In the current era of emerging technologies such as deep learning, artificial intelligence, multimedia data analysis, Internet of Things (IoT), the significant role of vibration analysis can be seen in biomedical, nonlinear dynamics, and control engineering. Despite the numerous applications, lots of challenges and issues are still needed to address like nonlinearity, fault diagnosis, vibration spectrum analysis, and biomedical data monitoring and processing. The aim of this Special Issue is to cover and address all the aforesaid challenges and issues and practical solutions for such emerging technologies. We have received 21 manuscripts in total for this Special Issue across the globe and after the rigorous review process, only 10 manuscripts have been accepted for publication. A brief overview of contributions to this Special Issue is as underneath.
\end{abstract}

Zhu Jia, and Ashutosh Sharma contributes an article entitled "An Exhaustive Study Engine Vibration Fault Analysis Based on Data Mining". In this article, the authors have proposed the method for the detection of rotating equipment faults by utilizing the vibration analysis. The spectral content is extracted and fed into the classifier like k-Nearest neighbors (KNN), backpropagation neural network BPNN, Sparse Representation Classifier (SRC), Support vector machine (SVM) and Random Forest (RF) for the type of failure prediction and analyze the unbalance condition (UNB), bearing faults (BDF), and broken rotor bars (BRB) faults. The RF classifier is better as compared to other classifiers in terms of accuracy, precision and recalls values by approximately $10.92 \%, 11.03 \%$ and $20.13 \%$ respectively.

Genling Huang, Lijuan Qiao, Shaweta Khanna, Pljonkin Anton Pavlovich, and Sandeep Tiwari contributes an article entitled "Research on Fan Vibration Fault Diagnosis based on Image Recognition". In this paper, a novel image recognition technique is proposed for vibration-based fault diagnosis using the spectrum images of the vibration signals. Artificial Neural Network (ANN) classifier yields better performance in terms of various performance parameters. The percentage improvement in accuracy for ANN classification methods over Support Vector Machine (SVM), k-Nearest Neighbours (kNN) and Random Forest Ensemble (RFE) methods are $10.01 \%, 4.51 \%$ and $2.01 \%$ respectively. Effectiveness of the proposed image recognition-based technique is compared with the state-of-the-art methods.

Xiaohong Zhang, Min Lin and Shalini Sharma, Shaweta Khanna, and Pljonkin Anton Pavlovich contributes an article entitled "Optical Fiber Deformation and Vibration Monitoring at Distinct Data Rates with Distinct Photo-Detectors Devices". In this article, two different methodologies for optical fiber deformation aiding vibration monitoring have been proposed. One is using the pin diode as a photo-detector device and other is manifesting the avalanche photodetector. It is found that pin diode and avalanche photodiode are exhibiting a quality factor of 40.5944 and 43.3523 at $30 \mathrm{Gbps}$, respectively. It has been concluded that use of avalanche photodiode at high bit rates is utilized.

Yubin Liu, Weiying Ding, Yufen Feng, and Yuxiu Guo contributes an article entitled 
"Ensembled Mechanical Fault Recognition System Based on Deep Learning Algorithm". In this paper, a combination of feature selection with Artificial Intelligence (AI) algorithm is presented for the mechanical fault recognition to deal with smart machine tools. Here, authors propose a Convolutional Neural Network $(\mathrm{CNN})$ based fault recognition and classification framework that uses the combination of feature extraction, feature vector decomposition using Empirical Mode Decomposition (EMD) and deep neural network (DNN) for recognising the different fault states of the rotating machinery. The proposed fault recognition and classification method performs better in terms of all the parameters yielding $99.01 \%$ accuracy with respective cross-entropy loss of 0.325 and time complexity of 18 mins and 31 seconds. The comparative shows that the maximum improvement of $12.29 \%$ is attained in terms of accuracy for the proposed fault recognition method.

Wei Wang, and Yu Shao contributes an article entitled "Building Vibration Monitoring Based on Digital Optical Cameras". In this paper, the vision-based monitoring with the sources of errors is presented. The PSNR and Structural Similarity are analysed for the performance comparison. The MAPE and MAE values obtained by the presented technique are less for all the signal samples. The proposed method outperforms the existed technique by $3.21 \%$ to $44.02 \%$ in form of MAPE. The proposed method outperforms well with the percentage improvement by $33.93 \%$ and $42.43 \%$ in terms of MAE.

YiYuan Cheng, Mingyang Su, Ming Hui,Wei Liu, and Yangbing Zheng contributes an article entitled "Mathematical simulation of adaptive vector finite element method for the analysis of electromagnetic vibration spectrum field response". This article realizes a three-dimensional adaptive vector finite meta-acting algorithm of controlled source electromagnetic vibration spectrum (CSEM) field to address 3D meshing for the simulation of terrain fluctuations and complex electrical anomalies. The two approaches analysed in this article are CSEM onedimensional positive algorithm and finite meta-method. The validity of the proposed algorithm is verified through numerical simulation of one-dimensional and three-dimensional models. The experimental analysis shows the ability and effectiveness of the algorithm to simulate the electromagnetic vibration spectrum field of complex geoelectrical structure.

Vilor L. Zakovorotny, and Valery E. Gvindjiliya contributes an article entitled "Self-organization and evolution in dynamic friction systems". This paper deals with the mathematical modeling of the processes of self-organization in a dynamic friction system in combination with the analysis of the equilibrium of the system, its stability, and the manifolds formed in the vicinity of the equilibrium. It is shown that the proposed mathematical models are integro-differential functionally coupled equations that describe self-organization. The study and experiments show that measurement of parameters available for estimation of some coordinates in the dynamic friction system allows one to solve the problem of non-decoupled dynamic monitoring of the state of friction units and opens new ways of improvement of friction units.

Anna Diligenskaya, and Alexandr Samokish contributes an article entitled "Parametric Identification of Technological Thermophysics Processes Based on Neural Network Approach". In this paper, the inverse problem of technological thermophysics under the influence of disturbing factors has been studied. The proposed solution combines the advantages of an accurate analytical method, and artificial intelligence methods. The attained results confirm the possibility of obtaining adequate solutions to the inverse problems of thermal conductivity with the intensity of the measurement noise in the range of $0-15 \%$. In the investigated class of solutions, the error in approximating the temperature state can be up to $2-5 \%$, and the error in restoring the unknown characteristic can be up to $7-10 \%$.

Hongyan Gu, Huimin Liang, Guoyun Tong, Fang Liu, Yu Liu, Xing Liu, Zhen Jia, and John Paul contributes an article entitled "Research on Vibration Mechanism and Control Technology of Building Structure Under Earthquake Action". In this article author develops a novel dynamically optimized and decentralized mechanism using the PID controller for the selfregulation of conventional PID controller-based method. The proposed simulated model provides 95.54\% earthquake tracking precision and can be used for developing the earthquake protective 
schemes for the adequate survivability of tall building structures as well as to safeguard the human occupant in it.

Anh Son Tran, Anh Huy Vo, Diep Cong Thanh Tu, and Ha Quang Thinh Ngo contributes an article entitled "Dynamical Analysis and Validation of Motion Control by Filtering Performance for Aerial Robotic System in Monitoring Agriculture". In this paper, the research topic related to Autonomous Aerial System (AAS) model to indicate the filtering effects in the agricultural application for making an alternative solution is presented. By utilizing various sensing devices such as gyroscope, accelerometer and compass in real-world hardware, the experimental results could evaluate more precise and efficient design. The findings of this study are to (1) propose the model of AAS and proper filters, (2) launch the verified process and calibration, and (3) demonstrate the competitive performance among filters.

We hope that the quality research work published in this special issue will be able to serve the concerned science, environment, and technology. The Guest Editors are thankful to the authors and reviewers who contributed to this special issue with their scientific work and useful comments, respectively. 